S.R. Mishra, Mohammad Mainul Hoque*, B. Mohanty, and N.N. Anika

\title{
Heat transfer effect on MHD flow of a micropolar fluid through porous medium with uniform heat source and radiation
}

https://doi.org/10.1515/nleng-2017-0126

Received October 3, 2017; revised February 4, 2018; accepted March 17, 2018.

\begin{abstract}
The present study examines the effect of heat transfer on electrically conducting MHD micropolar fluid flow along a semi-infinite horizontal plate with radiation and heat source. The uniform magnetic field has applied along the principal flow direction. The obtained governing equations have been converted into a set of dimensionless differential equations and then numerically solved by using a well-known Runge-Kutta method with shooting technique. The velocity, microrotation, and temperature distribution are presented for various physical parameters. The numerical values of skin friction and Nusselt numbers at the plates are shown in tabular form, and the obtained results are compared with the results of a previous study. It has been found that the magnetic parameter increases the velocity profile whereas the boundary layer thickness reduces due to the inclusion of coupling parameter and inertia effect. The presence/absence of magnetic parameter and coupling parameter enable to enhance the angular velocity profile while it is worth to note that the backflow has generated in the vicinity of the plate.
\end{abstract}

Keywords: MHD, micropolar fluid, porous medium, heat transfer, radiation

S.R. Mishra, B. Mohanty, Department of Mathematics, Institute of Technical Education and Research, Siksha 'O' Anusandhan University Odisha, Bhubaneswar-751030, India

*Corresponding Author: Mohammad Mainul Hoque, Discipline of Chemical Engineering, School of Engineering, University of Newcastle, NSW 2308, Australia, E-mail: mohammadmainul.hoque@uon.edu.au

N.N. Anika, Discipline of Mechanical Engineering, School of Engineering, University of Newcastle, NSW 2308, Australia

\section{Introduction}

The knowledge of micropolar fluids past a porous medium has significant practical applications across a wide range of areas namely polymer blends, porous rocks, alloys, foams and aerogels, microemulsions etc. One such application is in the field of lubrication, since the gap or the clearance in a bearing may be comparable to the average grain or molecular size of a non-Newtonian lubricant. For example, in batch mixers, sodium, which displays non-Newtonian characteristics, may be used both as a heat transfer agent and as a lubricant in the journal bearing supporting the mixing screw. The theory of micropolar fluid consisting of two different effects such as microrotational and micro-inertia, first proposed by Eringen [1]. The proposal led to the attention of the researchers in the magneto-hydrodynamic (MHD) micropolar fluid past in a porous medium due to its vast applications in engineering problems namely geothermal, energy extractions, oil exploration and the boundary layer control in the field of aerodynamics.

Many studies have examined the effect of heat transfer and magnetic field MHD micropolar fluid through a porous medium with different flow geometry such as a vertical plate, semi-infinite plate, channels etc. [2-6]. Raptis and Kafousias [7] and Kim [8] investigated the influence of magnetic field on two-dimensional steady incompressible flow confined in an infinite and moving vertical porous plate, respectively. Kim [8] reported that the velocity distributions of micropolar fluids are comparable with the Newtonian fluids. Additionally, it has shown that the surface skin friction decreased with increase in the moving vertical plate velocity. Beg et al. [9] studied the effect of heat generation/absorption on MHD free convection flow from a sphere to non-Darcian porous medium wherein the mathematical problem has been solved using network simulation method. There are some studies available in the open literature where the non-Newtonian fluid in a porous medium have investigated with different flow geometry [10-16]. Takhar et al. [17] have studied the unsteady three-dimensional MHD-boundary-layer 
flow due to the impulsive motion of a stretching surface. Baag et al. [18] investigated the MHD boundary layer flow over an exponentially stretching sheet past a porous medium with the uniform heat source. Mixed convection from a vertical surface embedded in a porous medium saturated with a non-Newtonian nanofluid has studied by Gorla et al. [19]. Chamkha et al. [20] has presented the non-similar solutions for mixed convection along a wedge embedded in a porous medium saturated by a non-Newtonian nanofluid. Similarity solutions for MHD thermosolutal Marangoni convection over a flat surface in the presence of heat generation or absorption effects have observed by Mudhaf and Chamkha [21]. RamReddy et al. [22] has examined soret impact on mixed convection flow in a nanofluid under convective boundary condition. Ramesh et al. [23] has presented the magnetohydrodynamic flow of a non-newtonian nanofluid over an impermeable surface with heat generation/absorption. Chamkha and Rashad [24] have observed the MHD forced convection flow of a nanofluid adjacent to a nonisothermal wedge. Recently, Raju et al. [25, 26] studied the radiation effect of Casson fluid over a moving wedge filled with gyrotactic microorganism and Carreau nanofluid over a cone packed with alloy nanoparticles, respectively. A molecular dynamics study on transient non-Newtonian MHD Casson fluid flow dispersion over a vertical radiative cylinder with entropy heat generation has studied by Reddy et al. [27]. The studies mentioned above concluded that the uniform magnetic field enables to control the heat generation of electrically conducting fluid.

The viscous dissipation is one of the significant parameters in fluid dynamics and usually very challenging to incorporate into the mathematical model. However, many researchers studied the effect of viscous dissipation on hydromagnetic flow through a saturated porous medium and proposed a different mathematical model based on many ways [28-34]. Chamkha et al. [35] have presented non-Darcy natural convection flow for nonNewtonian nanofluid over cone saturated in a porous medium with uniform heat and volume fraction fluxes. Gorla and Chamkha [36] has observed natural convective boundary layer flow over a horizontal plate embedded in a porous medium saturated with a nanofluid. Al-Hadhrami et al. [37] proposed a new viscous dissipation model for a porous medium, which is most suitable for most practical applications. It has found that the porous medium enables to enhance the thermal performance when the aspect ratio of the channel or the thermal conductivity of the channel matrix increased.

In the last few years, many researchers are devoted to investigating the unsteady micropolar fluid through a porous medium in the presence or absence of a uniform magnetic field [38, 39]. Mohanty et al. [40] numerically studied the effect of heat and mass transfer on MHD micropolar fluid using Runge-Kutta fourth order method followed by shooting technique. It has found that the Lorentz force produced by the magnetic field which enables the resistance to momentum field in the presence/absence of porous matrix. Using the similar numerical techniques (Mohanty et al. [40]), the effect of heat source parameter on electrically conducting micropolar fluid has investigated by Mishra et al. [41]. Due to the presence/absence of heat source parameter, the two layers variation has observed in the thermal boundary layer. Later, Tripathy et al. [42] studied the effect of chemical reaction on MHD micropolar fluid considering moving the vertical porous plate. The thermal radiation significantly influences the temperature distribution and heat transfer of electrically conducting micropolar fluid. But here, it is noted that there are very few publications available in the literature which analysed the effect of thermal radiation and heat transfer of micropolar fluid and demands more systematic investigation in future. Rashedi et al. [43] proposed analytical solutions based on homotopy analysis method (HAM) for micropolar fluid past through a porous medium.

In the analysis of flow through a porous medium, Darcy's law usually has been considered to be the fundamental equation (Muskat [44]; Collins [45]). The principle of Darcy's law states that the velocity components are directly proportional to the pressure gradient where the convective acceleration term of fluid does not exist. Hence, this law is only valid for low-speed flows. The force of the fluid which is also proportional to the velocity components may deviate from the Darcy drag force (Bear [46]). The generalisation proposed by Brinkman considered the convective force. To study the flow through a highly porous medium (such as fibres) the generalised Darcy law is recommended. Hence, in the present study, generalised Darcy law has been used to account for the porosity of the medium. Consequently, the novelties of the present study placed down as follows:

1. The momentum transport equation has been modified by inclusion of two terms i.e. $\frac{\sigma B_{0}{ }^{2} \varphi}{\rho}\left(U_{0}-u\right)$ magnetic parameter and $C \varphi\left(U_{0}{ }^{2}-u^{2}\right)$ (Non-Darcian) which account for the effect of permeability of the medium on the flow phenomena.

2. The heat energy equation has been generalised by considering the uniform heat source/sink, not taken care of by Rashidi et al. [43] which affect the heat transport phenomena. 
Motivated by these applications the present study explores the effects of the magnetic parameter, heat source/sink parameter and inertia effect of micropolar fluid by modifying the momentum and energy equations, which primarily constitutive the flow model of any liquid. To achieve this aim, we considered the electrically conducting MHD micropolar flow past through a semiinfinite horizontal plate. The porous medium with variable suction velocity and viscous dissipation are discussed in the analysis. The effect of viscous dissipation is modelled by following the suggestion proposed by Al-Hadhrami et al. [37]. It is worth to mention that, the proposed mathematical model is highly non-linear, hence only approximate numerical solution is possible in contrast to the analytical solution. Finally, the obtained results are compared with the analytical results reported by Rashidi et al. [43] as a particular case.

\section{Mathematical formulation}

A steady two-dimensional MHD flow of a micropolar fluid through a porous medium past a semi-infinite horizontal plate in the presence of uniform heat source was considered in the present study. The effect of radiation was analysed by modifying the energy equation. The semi-infinite horizontal plate is placed along the $x$-direction, and $y$-axis is perpendicular to it. We assumed that a uniform transverse magnetic field of strength $B_{0}$ employed to the principle direction of flow. The transverse magnetic field can be negligible due to the small magnetic Reynold number. A schematic of the flow geometry along with coordinate system is shown in Fig. 1.

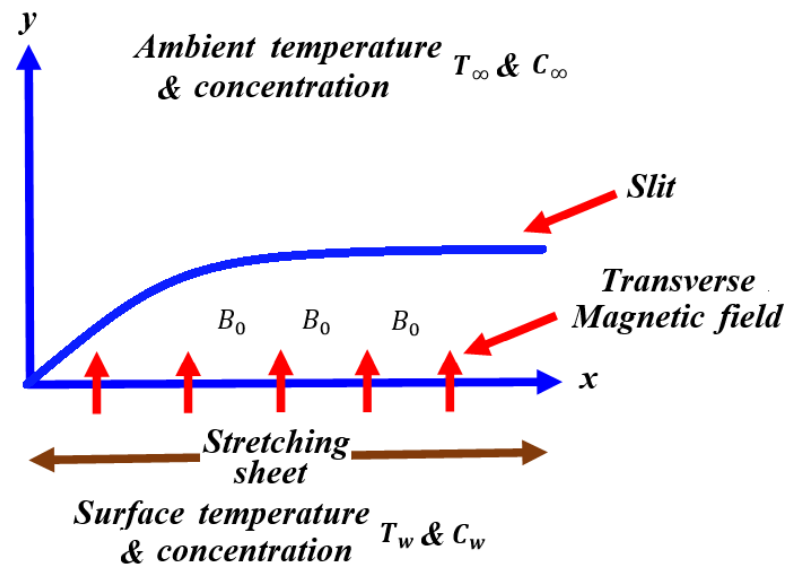

Fig. 1: Schematic of flow geometry
All the physical parameters in the proposed model have been independent along the $\mathrm{x}$-direction as we considered an infinite plate along the vertical direction ( $\mathrm{x}$ direction). To investigate the effects of heat absorption, a heat source parameter has been applied to the flow. Hence, the set of governing equations of the problem can be expressed as:

$$
\begin{gathered}
\frac{\partial u}{\partial x}+\frac{\partial v}{\partial y}=0 \\
u \frac{\partial u}{\partial x}+v \frac{\partial v}{\partial y}=v \frac{\partial^{2} u}{\partial y^{2}}+k_{1} \frac{\partial N}{\partial y} \\
+\frac{\sigma B_{0}{ }^{2} \varphi}{\rho}\left(U_{0}-u\right)+\frac{v \varphi}{K_{p}{ }^{*}}\left(U_{0}-u\right)+C \varphi\left(U_{0}{ }^{2}-u^{2}\right) \\
G_{1} \frac{\partial^{2} N}{\partial y^{2}}-2 N-\frac{\partial u}{\partial y}=0
\end{gathered}
$$

$$
u \frac{\partial T}{\partial x}+v \frac{\partial T}{\partial y}=\frac{k}{\rho C_{p}} \frac{\partial^{2} T}{\partial y^{2}}-\frac{1}{\rho C_{p}} \frac{\partial q_{r}}{\partial y}+\frac{S^{\prime}}{\rho C_{p}}\left(T-T_{\infty}\right)
$$

By assuming Rosseland approximation, the radiative heat flux is taken as

$$
q_{r}=-\frac{4 \sigma^{\star}}{3 k^{\star}} \frac{\partial T^{4}}{\partial y},
$$

where $\sigma^{\star}$ is the Stefan-Boltzmann constant and $k^{\star}$ is the mean absorption coefficient. Assuming that the temperature differences within the fluid in the boundary layer are sufficiently small, we can express the term $T^{4}$ as a linear function of temperature. Hence, using expanding $T^{4}$ in a Taylor series about $T_{w}$ and neglecting higher-order terms, we obtain. Then, $q_{r}$ can be expressed as

$$
q_{r}=-\frac{16 \sigma^{\star} T_{\infty}^{3}}{3 k^{\star}} \frac{\partial T}{\partial y}
$$

The associated boundary conditions of the present mathematical problem can be defined as follows:

$$
\begin{array}{ll}
u=0, v=0, N=0, T=T_{w} & \text { at } y=0 \\
u=U_{0}, N=0, T=T_{\infty} & \text { as } y \rightarrow \infty
\end{array}
$$

\section{Solution of the problem}

The following non-dimensional variables have been used to achieve the dimensionless form of the governing equations:

$$
\left.\begin{array}{l}
\psi(x, y)=\left(2 v U_{0} x\right)^{\frac{1}{2}} f(\eta) \\
N=\left(\frac{U_{0}}{2 v x}\right)^{\frac{1}{2}} U_{0} g(\eta) \\
\eta=\left(\frac{U_{0}}{2 v x}\right)^{\frac{1}{2}} y \\
\theta=\frac{T-T_{\infty}}{T_{w}-T_{\infty}}
\end{array}\right\}
$$


Using Eq. (6) into the Eqs. (2) - (4), the nondimensional governing equations can be written as:

$$
\begin{gathered}
f^{\prime \prime \prime}+f f^{\prime \prime}+\Delta g^{\prime}+\left(M+\frac{1}{K_{p}}\right)\left(1-f^{\prime}\right)+F\left(1-f^{\prime 2}\right)=0 \\
G g^{\prime \prime}-2\left(2 g+f^{\prime \prime}\right)=0 \\
(3 R+4) \theta^{\prime \prime}+3 R \mathrm{P}_{r} f \theta^{\prime}+S \mathrm{P}_{r} \theta=0
\end{gathered}
$$

Hence, the boundary conditions (Eq. 5) can be expressed as:

$$
\begin{array}{ll}
f(\eta)=0, f^{\prime}(\eta)=0, g(\eta)=0, \theta(\eta)=1 & \text { at } \eta=0 \\
f^{\prime}(\eta) \rightarrow 1, g(\eta) \rightarrow 0, \theta(\eta) \rightarrow 0 & \text { as } \eta \rightarrow \infty
\end{array}
$$

where,

$$
\begin{aligned}
& \mathrm{P}_{r}=\frac{\mu C_{p}}{k}, \Delta=\frac{k_{1}}{v}, G=\frac{G_{1} U_{0}}{v x}, R=\frac{k^{*} k}{4 \sigma^{*} T_{\infty}^{3}}, K_{p}=\frac{2 \varphi v x}{K_{p}^{*} U_{0}}, \\
& F=2 \varphi C x, M=\frac{\sigma B_{0}^{2} \varphi x}{\rho} k_{1}=\rho \kappa, v=(\mu+\kappa) / \rho, S=\frac{2 S^{\prime} x}{\rho C_{p} U_{0}},
\end{aligned}
$$

In the present study, the local skin friction coefficient $\left(C_{f}\right)$, the local wall couple stress $\left(M_{w x}\right)$ and the local heat transfer coefficient $\left(N u_{x}\right)$ can be defined by the following relation:

$$
\begin{gathered}
C_{f}=\frac{\tau_{w}}{\rho U_{0}{ }^{2} / 2}, \quad \tau_{w}=\left[(\mu+\kappa) \frac{\partial u}{\partial y}+\kappa N\right]_{y=0}=\frac{2 U_{0} X}{v} f^{\prime \prime}(0) \\
M_{w x}=G_{1}\left(\frac{\partial N}{\partial y}\right)=\frac{U_{0} X}{v} g^{\prime}(0) \\
N u_{x}=\frac{x q_{w}}{k\left(T_{w}-T_{\infty}\right)}=-\frac{v}{U_{0} x} \theta^{\prime}(0), q_{w}=-k\left(\frac{\partial T}{\partial y}\right)_{y=0}
\end{gathered}
$$

\section{Numerical solution}

The set of coupled non-linear differential Eqs. (7) - (9) have been solved numerically using robust shooting method with Runge-Kutta scheme and considering the boundary conditions mentioned in Eq. (10). By following the way of superposition the Eq. (7) - (9) were expressed as $1^{\text {st }}$ order simultaneous equations of seven unknown variables. We considered that $y_{1}=f, y_{2}=f^{\prime}, y+3=f^{\prime \prime}, y_{4}=g, y_{5}=$ $g^{\prime}, y_{6}=\theta, y_{7}=\theta^{\prime}$. Hence, the Eqs. (7) $-(9)$ can be written as a set of ordinary differential equations. i.e.

$$
y_{3}^{\prime}=-y_{1} y_{3}-\Delta y_{5}-\left(M+\frac{1}{K_{p}}\right)\left(1-y_{2}\right)-F\left(1-y_{2}^{2}\right)
$$

$$
y_{5}^{\prime}=\frac{2}{G}\left(2 y_{4}+y_{3}\right)
$$

$$
y_{7}^{\prime}=\frac{-P_{r}}{4+3 R}\left[3 R y_{1} y_{7}+S y_{6}\right]
$$

with the boundary conditions

$$
\left.\begin{array}{c}
y_{1}(0)=0, y_{2}(0)=0, y_{2}(\infty)=1, y_{4}(0)=0, \\
y_{4}(\infty)=0, y_{6}(0)=1, y_{6}(\infty)=0
\end{array}\right\}
$$

The simulation has been repeated for a considerable value of $\eta_{\infty}$ up to two consecutive values of $f^{\prime \prime}(0), f^{\prime \prime \prime}(0), g^{\prime \prime}(0)$ and $\theta^{\prime}(0)$ only diverse when the digit indicates the limit of the boundary towards $\eta$. For a particular set of parameters, the final value of $\eta_{\infty}$ is chosen based on the limit $\eta \rightarrow \infty$. The simulation has repeated till the accuracy of $10^{-6}$ is attended.

\section{Results and discussion}

A numerical approach such as Runge-Kutta method with shooting technique has been used to solve the non-linear self-similar governing equations (2) to (4), where Eq. (5) denote the two-point boundary value problem (BVP). To convert the BVP into IVP, we assign some guessed value with unknown initial conditions and initiate the process of computation. The step size of the current simulation has fixed at $\eta=0.001$. Here it is noted that the present solution affected by two additional parameters namely magnetic parameter $(M)$ in the momentum equation and uniform heat source/sink parameter in the energy equation (not considered by Rashidi et al. [43]).

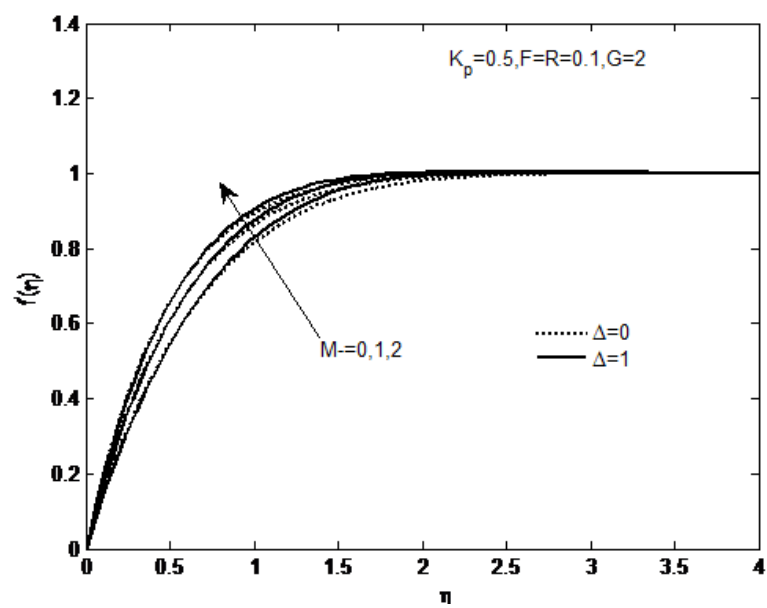

Fig. 2: Velocity profile for different values of $M$ and $\Delta$ 


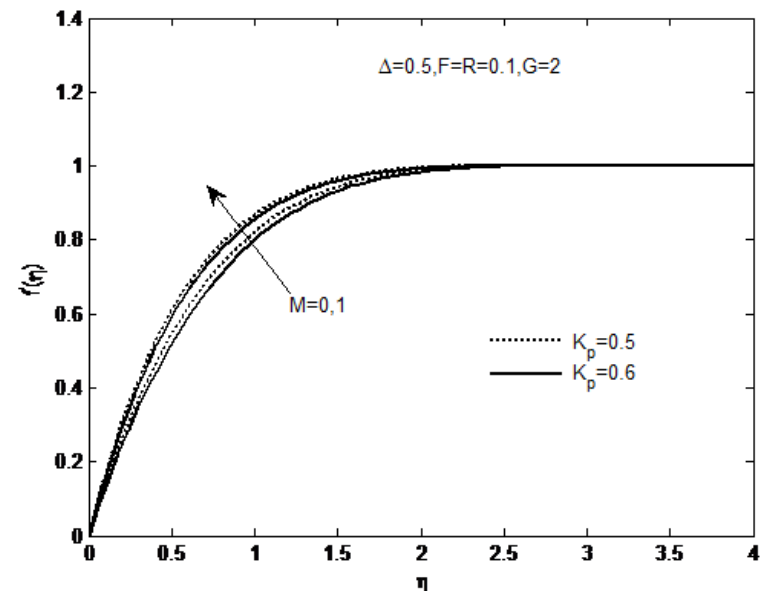

Fig. 3: Velocity profile for different values of $M$ and $K_{p}$

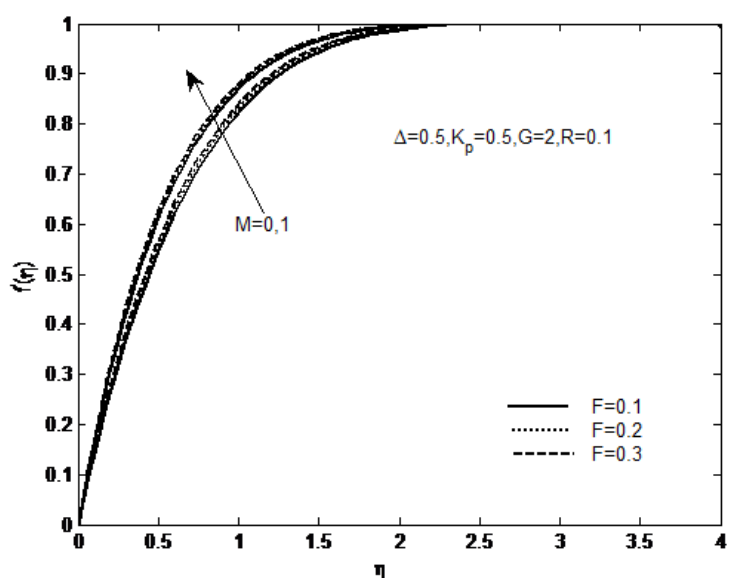

Fig. 4: Velocity profile for different values of $M$ and $F$

Figs. 2 - 4 reveal the velocity profile for different physical parameters such as magnetic parameter $(M)$, the porous parameter $\left(K_{p}\right)$, coupling parameter $(\Delta)$, radiation parameter $(R)$ and microrotation parameter $(G)$. The illustration of microrotation profile has shown in Figs. 5 - 7 with the inclusion of above physical parameter. The effect of heat source/sink in the absence/presence of and with radiation parameter have been discussed in Figs. 8 -10. Fig. 2 exhibits the impact of the magnetic parameter, $M$ on the velocity distribution in the absence/presence of coupling parameter and the fixed values of $K_{p}=0.5, F=R=0.1$ and $G=2$, respectively. It is observed that the velocity profile enhanced with the increase of magnetic parameter in the absence/presence of coupling parameter $(\Delta=0$ and $\Delta=1)$. The higher value of the magnetic parameter $(M=2)$ increases the velocity distribution, hence, the velocity boundary layer thickness decreased. It is because the magnetic field applied along to the normal of flow direction. This magnetic field gives rise to a resistive force and slows down the movement of the fluid. The velocity profile was remaining invariant from $\eta \geq 2$.

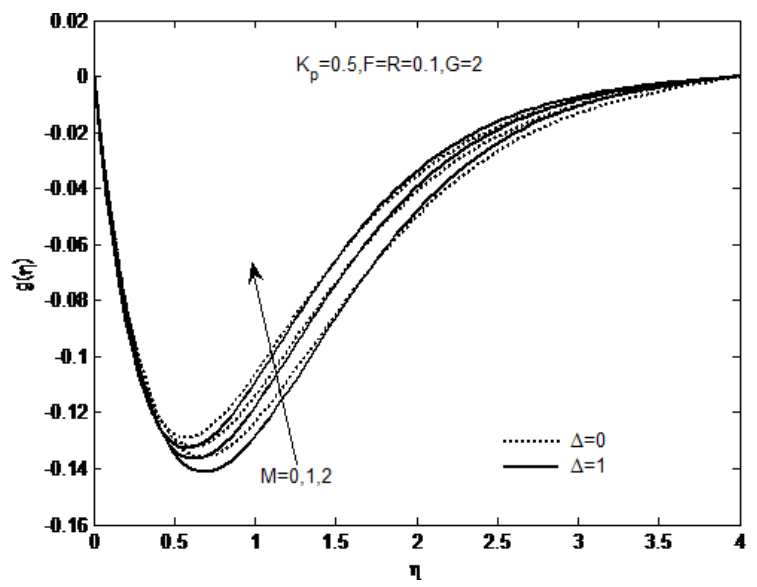

Fig. 5: Angular velocity profile for different values of $M$ and $\Delta$

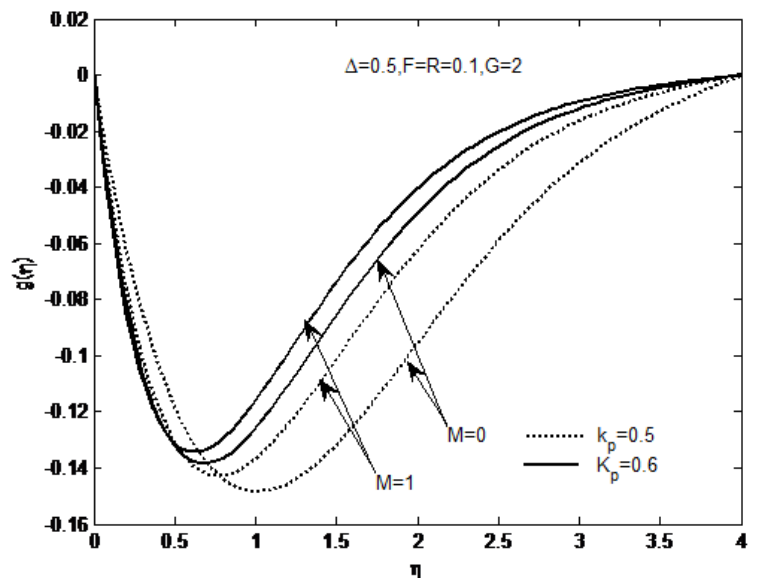

Fig. 6: Angular velocity profile for different values of $M$ and $K_{p}$

The velocity profile for the dimensionless parameters $M$ and $K_{p}$ is shown in Fig. 3 with the fixed values of $\Delta=$ $0.5, F=R=0.1$ and $G=2$. The velocity profile observed to decelerates in the boundary layer in the presence/absence of magnetic parameters due to the porous matrix. It is also noted that the reverse effect appears to be right in the case of the magnetic parameter. Fig. 4 illustrates the effect of $M$ and $F$ on velocity profile. It is clear that the increase in microinertia coefficient increases the velocity profile at all points in the flow domain for both presence/absence of magnetic parameter. The present result is in good agreement with the outcome of Rashidi et al. [43] by withdrawing the magnetic parameter, i.e. $M=0$ from the velocity profile. 
Fig. 5 exhibits the effect ofand coupling parameter $(\Delta)$ on angular velocity profile with fixed values of other parameters such as $K_{p}=0.5, F=R=0.1$ and $G=2$. It is clear that in the presence/absence of $M$ and $\Delta$, angular velocity profile increases and the backflow is generated in the vicinity of the plate after this it meets the boundary conditions. It has appeared that the magnetic field retards the angular velocity profile with coupling parameter. The effect of $M$ and $K_{p}$ on angular velocity is exhibited in Fig. 6. It is interesting to note that magnetic field enhances the profile whereas, $K_{p}$ retards it. A similar observation has well marked that the backflow occurs in the boundary layer.

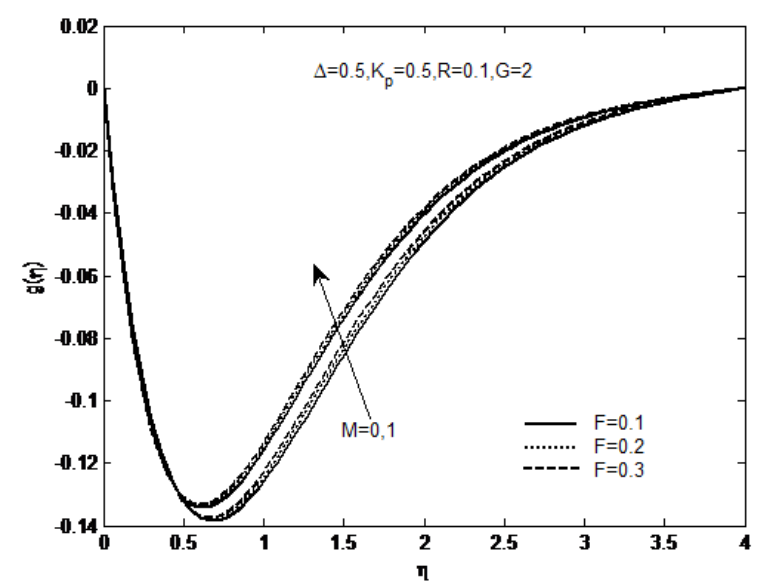

Fig. 7: Angular velocity profile for different values of $M$ and $F$

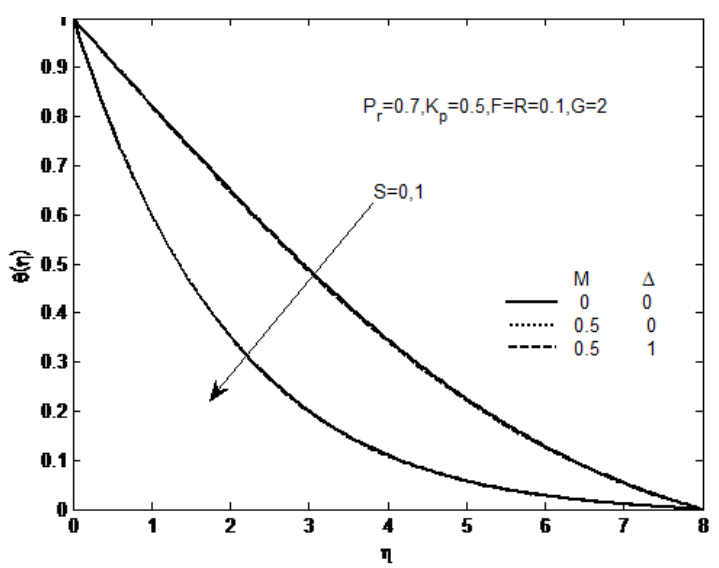

Fig. 8: Temperature profile for different values of $S, \Delta$ and $M$

Fig. 7 illustrates the effect of $M$ and $F$ on angular velocity profile with the fixed values of the pertinent parameters $K_{p}=0.5=\Delta, R=0.1$ and $G=2$. It is remarked that the angular velocity has linear behaviour within the flow domain $\eta<0.5$ and after that, it rises to meet the boundary conditions. We observed that the increase in microinertia coefficient had enhanced the profile at all points in the boundary layer. Due to the Lorentz force (often treated as a resistive force), a reduction in the velocity observed whereas the reverse effect has encountered with the higher values of $M$. The critical aspect of the present study is the effect of uniform heat source on the temperature profile in the presence/absence of the $M, \Delta, K_{p}$ and $R$ parameters with the fixed values of other pertinent parameters.

From Fig. 8, it is remarked that increase in heat source parameter retards the temperature profile at all points in the presence of $M$ and $\Delta(M=0.5$ and $\Delta=1)$. In contrast for and, the maximum temperature has been observed at the boundary-layer which is parabolic in nature. It is because the resistive lorenze force which retards a certain amount of energy stored up in the thermal boundary layer, therefore, the temperature profile increased.

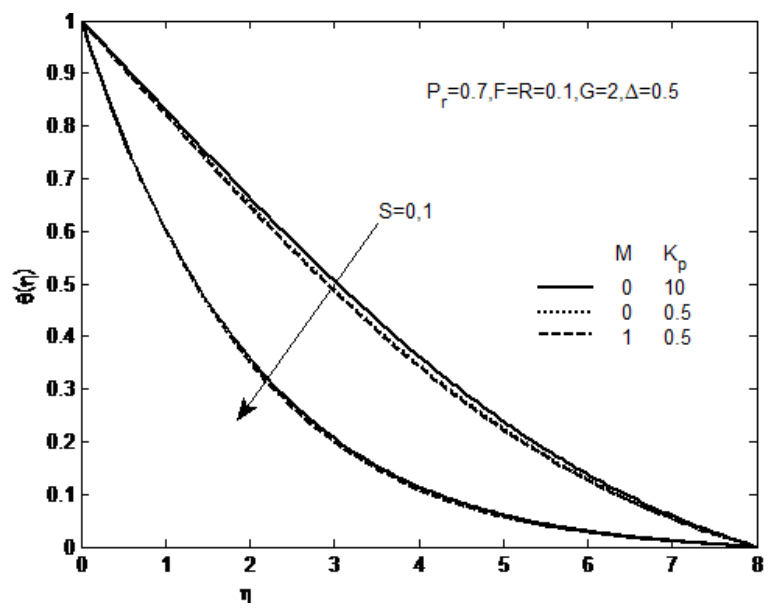

Fig. 9: Temperature profile for various values of $S, M$ and $K_{p}$

Fig. 9 illustrates the effect of the uniform heat source in the presence/absence of $M$ and $K_{p}$. It is evident that growth in $M$ and $K_{p}$ the temperature profile irrespectively decelerates for different values of the heat source. When $M=0, S=0$ and $K_{p}$ (without porous matrix) the profile attaining the maximum value and tends to meet the boundary conditions for $\eta \rightarrow \infty$. The thermal radiation effect has been observed in both the presence/absence of heat source and shown in Fig. 10. There two-layer variations in temperature profile have been remarked for $S=0$ and $S=1$ at different values of radiation parameter $(R)$. From Fig. 10, it can be observed that the temperature decreases in the thermal boundary layer with an increase in $R$ and $S$. The present results make a good agreement with the outcome of Rashidi et al. [23] by withdrawing the parame- 


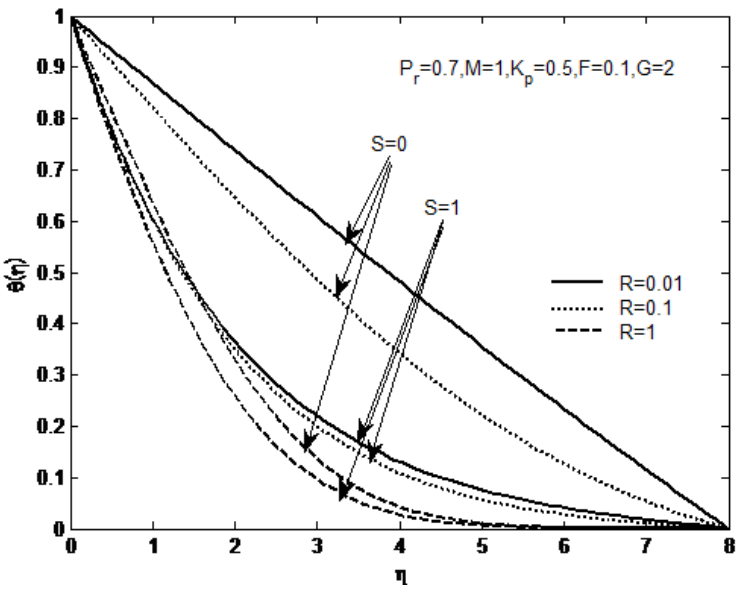

Fig. 10: Temperature profile for different values of $S$ and $R$

ter $S(S=0)$ from the energy equation. Thermal radiation emitted by a hot body depends not only on its temperature but also on the material of the body, its shape and the nature of its surface.

The flow characteristics at the boundary surface are vital in determining the flow stability and hence calculation of skin friction, wall couple stress and Nusselt number are essential. The numerical values of aforesaid parameters have been shown in Table 1 for different pertinent parameters. It can appear that the skin friction and rate of heat transfer increases with the increase of magnetic parameter while the reverse effect has been observed for couple stress. The inclusion of porous matrix opposes the flow and heat transfer aspect resulting in retardation in skin friction coefficient and Nusselt number, but couple stresses enhanced it. In the unsteady case, the presence of heat source parameter increases the values of $f^{\prime \prime}(0), g^{\prime}(0)$ and $-\theta^{\prime}(0)$.

\section{Conclusions}

In the present study, the effect of heat transfer on an electrically conducting MHD micropolar fluid through a porous medium has been analysed considering radiation and uniform heat source. The present simulations results highlighted the following facts:

1. The velocity profile enhances at all points in the boundary layer with an increase in the magnetic parameter for the absence/presence of coupling parameter.

2. The velocity distribution increases with increase in microinertia coefficient for both presence/absence of magnetic parameter.
3. The temperature distributions in the thermal boundary layer follow a declining trend as the radiation and source parameter increases.

4. The increase in magnetic parameter and porous parameter decelerates the temperature profile irrespectively for the values of the heat source parameter.

5. The presence/absence of magnetic parameter and coupling parameter enable to enhance the angular velocity profile while it is worth to note that the backflow has generated in the vicinity of the plate.

6. In the absence of heat source, i.e. $S=0$, present result is in good agreement with Rashidi et al. [43].

\section{Nomenclature}

\begin{tabular}{ll}
\hline$B_{0}$ & applied magnetic field \\
$C_{f x}$ & local skin friction co-efficient \\
$C_{p}$ & specific heat at constant pressure \\
$R$ & radiation parameter \\
$F$ & Inertia coefficient parameter \\
$f$ & dimensionless stream function \\
$G$ & microrotation parameter \\
$K_{p}$ & local porous parameter \\
$k$ & thermal conductivity \\
$K_{p}{ }^{*}$ & permeability of the porous medium \\
$M$ & magnetic field parameter \\
$M_{S x}$ & local wall couple stress \\
$N_{u x}$ & local Nusselt number \\
$P_{r}$ & Prandtl number \\
$Q_{0}$ & heat source coefficient \\
$R_{e x}$ & local Reynold number \\
$S$ & heat source parameter \\
$T$ & non-dimensional temperature \\
$t$ & non-dimensional time \\
$T_{\omega}$ & wall temperature of the fluid \\
$T_{\infty}$ & temperature of the fluid far away from the sheet \\
$U_{\omega}$ & sheet velocity \\
$(u, v)$ & velocity components \\
$(x, y)$ & cartesian coordinates \\
\hline$G r e e k$ & symbol
\end{tabular}

\begin{tabular}{ll}
\hline \multicolumn{2}{l}{ Greek symbol } \\
\hline$v$ & kinematics coefficient of viscosity \\
$\mu$ & coefficient of viscosity \\
$\sigma$ & electrical conductivity \\
$\Delta$ & coupling constant \\
$\rho$ & density of the fluid \\
$\tau_{w}$ & wall shear stress \\
$\theta$ & non-dimensional temperature \\
$\eta$ & similarity variable \\
\hline subscripts \\
\hline$\omega$ & condition at wall \\
$\infty$ & condition at free stream \\
\hline
\end{tabular}


Table 1: Skin friction, wall couple stress, Nusselt number for the values of $R=0.1, G=2$.

\begin{tabular}{cccccccc}
\hline$M$ & $K_{p}$ & $F$ & $S$ & $\Delta$ & $f^{\prime \prime}(0)$ & $g^{\prime}(0)$ & $-\theta^{\prime}(0)$ \\
\hline $\mathbf{0}$ & 0.5 & 0.1 & 0 & 0 & 1.520775 & -0.53477 & 0.271877 \\
$\mathbf{1}$ & 0.5 & 0.1 & 0 & 0 & 1.819147 & -0.57523 & 0.273121 \\
$\mathbf{1}$ & 0.6 & 0.1 & 0 & 0 & 1.725367 & -0.56327 & 0.272758 \\
$\mathbf{1}$ & 0.5 & 0.2 & 0 & 0 & 1.855385 & -0.58043 & 0.27328 \\
$\mathbf{1}$ & 0.5 & 0.1 & 1 & 0 & 1.819147 & -0.57523 & 0.517112 \\
$\mathbf{1}$ & 0.5 & 0.1 & 1 & 1 & 1.728685 & -0.57578 & 0.517155 \\
$\mathbf{2}$ & 0.5 & 0.1 & 1 & 1 & 1.987426 & -0.60527 & 0.517801 \\
$\mathbf{2}$ & 0.6 & 0.1 & 1 & 1 & 1.904827 & -0.5963 & 0.517605 \\
$\mathbf{2}$ & 0.5 & 0.2 & 1 & 1 & 2.019429 & -0.60923 & 0.517887 \\
\hline
\end{tabular}

\section{References}

[1] A.C. Eringen, Simple micro fluids, Int. J. Eng. Sci, 2 (1964) 205217.

[2] N.N. Anika, M.M. Hoque, S.I. Hossain, M.M. Alam, Thermal diffusion effect on unsteady viscous MHD micropolar fluid flow through an infinite vertical plate with Hall and ion-slip current, Procedia Engineering, 105 (2015) 160-166.

[3] J.I. Oahimire, B.I. Olajuwon, Effect of Hall current and thermal radiation on heat and mass transfer of a chemically reacting MHD flow of a micropolar fluid through a porous medium, Journal of King Saud University-Engineering Sciences, 26 (2014) 112-121.

[4] D. Prakash, M. Muthtamilselvan, Effect of radiation on transient MHD flow of micropolar fluid between porous vertical channel with boundary conditions of the third kind, Ain Shams Engineering Journal, 5 (2014) 1277-1286.

[5] R. Sharma, R. Bhargava, I.V. Singh, Combined effect of magnetic field and heat absorption on unsteady free convection and heat transfer flow in a micropolar fluid past a semi-infinite moving plate with viscous dissipation using element free Glalerkin method, Applied Mathematics and Computation, 217 (2010) 308-321.

[6] R. Tripathy, G. Dash, S. Mishra, M.M. Hoque, Numerical analysis of hydromagnetic micropolar fluid along a stretching sheet embedded in porous medium with non-uniform heat source and chemical reaction, Engineering science and technology, an international journal, 19 (2016) 1573-1581.

[7] A. Raptis, N. Kafousias, Heat transfer in flow through a porous medium bounded by an infinite vertical plate under the action of a magnetic field, International journal of energy research, 6 (1982) 241-245.

[8] Y.J. Kim, Unsteady MHD convection flow of polar fluids past a vertical moving porous plate in a porous medium, International Journal of Heat and Mass Transfer, 44 (2001) 2791-2799.

[9] O.A. Bég, J. Zueco, R. Bhargava, H.S. Takhar, Magnetohydrodynamic convection flow from a sphere to a non-Darcian porous medium with heat generation or absorption effects: network simulation, International Journal of Thermal Sciences, 48 (2009) 913-921.

[10] A. Chamkha, A. Aly, MHD free convection flow of a nanofluid past a vertical plate in the presence of heat generation or absorption effects, Chemical Engineering Communications, 198 (2010) 425-441.

[11] A.J. Chamkha, S. Abbasbandy, A. Rashad, K. Vajravelu, Radi- ation effects on mixed convection over a wedge embedded in a porous medium filled with a nanofluid, Transport in Porous Media, 91 (2012) 261-279.

[12] A.J. Chamkha, S. Abbasbandy, A. Rashad, K. Vajravelu, Radiation effects on mixed convection about a cone embedded in a porous medium filled with a nanofluid, Meccanica, 48 (2013) 275-285.

[13] Durgaprasad, P., Varma, S.V.K., Hoque, M.M. and Raju, C.S.K., Combined effects of Brownian motion and thermophoresis parameters on three-dimensional (3D) Casson nanofluid flow across the porous layers slendering sheet in a suspension of graphene nanoparticles, Neural Computing \& Applications (2018). https://doi.org/10.1007/s00521-018-3451-z

[14] R.S.R. Gorla, A. Chamkha, Free convection past a vertical plate embedded in a porous medium saturated with a nonNewtonian nanofluid, Journal of Nanofluids, 2 (2013) 297-302.

[15] R.S.R. Gorla, A. Chamkha, K. Ghodeswar, Natural convective boundary layer flow over a vertical cone embedded in a porous medium saturated with a nanofluid, Journal of Nanofluids, 3 (2014) 65-71.

[16] C.S.K. Raju, M.M. Hoque, S. Priyadharshini, B.J. Gireesha, Cross diffusion effects on magnetohydrodynamic slip flow of Carreau liquid over a slendering sheet with non-uniform heat source/sink, Journal of the Brazilian Society of Mechanical Science and Engineering, 40 (4) (2018), pp. 222.

[17] H.S. Takhar, A.J. Chamkha, G. Nath, Unsteady threedimensional MHD-boundary-layer flow due to the impulsive motion of a stretching surface, Acta Mechanica, 146 (2001) 59-71.

[18] S. Baag, S. Mishra, M.M. Hoque, N.N. Anika, Magnetohydrodynamic Boundary Layer Flow Over an Exponentially Stretching Sheet Past a Porous Medium with Uniform Heat Source, Journal of Nanofluids, 7 (2018) 570-576.

[19] Rama Subba Reddy Gorla, Ali Jawad Chamkha, A.M. Rashad, Mixed Convection from a Vertical Surface Embedded in a Porous Medium Saturated with a Non-Newtonian Nanofluid, Journal of Applied Fluid Mechanics, 6 (2013) 301-309.

[20] A. J. Chamkha, M. Rashad, R. Subba Reddy Gorla, Non-similar solutions for mixed convection along a wedge embedded in a porous medium saturated by a non-Newtonian nanofluid: Natural convection dominated regime, International Journal of Numerical Methods for Heat \& Fluid Flow, 24 (2014) 14711486.

[21] A. Al-Mudhaf, A.J. Chamkha, Similarity solutions for MHD thermosolutal Marangoni convection over a flat surface in the 
presence of heat generation or absorption effects, Heat and Mass Transfer, 42 (2005) 112-121.

[22] C. RamReddy, P. Murthy, A.J. Chamkha, A. Rashad, Soret effect on mixed convection flow in a nanofluid under convective boundary condition, International Journal of Heat and Mass Transfer, 64 (2013) 384-392.

[23] G. Ramesh, A.J. Chamkha, B. Gireesha, Magnetohydrodynamic flow of a non-Newtonian nanofluid over an impermeable surface with heat generation/absorption, Journal of nanofluids, 3 (2014) 78-84.

[24] A.J. Chamkha, A. Rashad, MHD forced convection flow of a nanofluid adjacent to a non-isothermal wedge, Computational Thermal Sciences: An International Journal, 6 (2014).

[25] C.S.K. Raju, M.M. Hoque, N.N. Anika, S.U. Mamatha, P. Sharma, Natural convective heat transfer analysis of MHD unsteady Carreau nanofluid over a cone packed with alloy nanoparticles, Powder Technology, 317 (2017) 554-562.

[26] C.S.K. Raju, M.M. Hoque, T. Sivasankar, Radiative flow of Casson fluid over a moviing wedge filled with gyrotactic microorganisms, Advanced Powder Technology, 28 (2017) 575-583.

[27] G.J. Reddy, B. Kethireddy, M. Kumar, M.M. Hoque, A molecular dynamics study on transient non-Newtonian MHD Casson fluid flow dispersion over a radiative vertical cylinder with entropy heat generation, Journal of Molecular Liquids, 252 (2018) 245262.

[28] A. Chamkha, Solar radiation assisted natural convection in uniform porous medium supported by a vertical flat plate, Journal of heat transfer, 119 (1997) 89-96.

[29] A. Chamkha, R.S.R. Gorla, K. Ghodeswar, Non-similar solution for natural convective boundary layer flow over a sphere embedded in a porous medium saturated with a nanofluid, Transport in Porous Media, 86 (2011) 13-22.

[30] A. Chamkha, A. Rashad, Natural convection from a vertical permeable cone in a nanofluid saturated porous media for uniform heat and nanoparticles volume fraction fluxes, International Journal of Numerical Methods for Heat \& Fluid Flow, 22 (2012) 1073-1085.

[31] A.J. Chamkha, C. Issa, K. Khanafer, Natural convection from an inclined plate embedded in a variable porosity porous medium due to solar radiation, International Journal of Thermal Sciences, 41 (2002) 73-81.

[32] R.S.R. Gorla, A. Chamkha, Natural convective boundary layer flow over a nonisothermal vertical plate embedded in a porous medium saturated with a nanofluid, Nanoscale and Microscale Thermophysical Engineering, 15 (2011) 81-94.

[33] R.S.R. Gorla, A.J. Chamkha, A.M. Rashad, Mixed convective boundary layer flow over a vertical wedge embedded in a porous medium saturated with a nanofluid: natural convection dominated regime, Nanoscale Research Letters, 6 (2011) 207.
[34] M.M. Hoque, M.J. Sathe, S. Mitra, J.B. Joshi, G.M. Evans, Comparison of specific energy dissipation rate calculation methodologies utilising 2D PIV velocity measurement, Chemical Engineering Science, 137 (2015) 752-767.

[35] A. Chamkha, S. Abbasbandy, A. Rashad, Non-Darcy natural convection flow for non-Newtonian nanofluid over cone saturated in porous medium with uniform heat and volume fraction fluxes, International Journal of Numerical Methods for Heat \& Fluid Flow, 25 (2015) 422-437.

[36] R.S.R. Gorla, A. Chamkha, Natural convective boundary layer flow over a horizontal plate embedded in a porous medium saturated with a nanofluid, Journal of Modern Physics, 2 (2011) 62.

[37] A. Al-Hadhrami, L. Elliott, D. Ingham, A new model for viscous dissipation in porous media across a range of permeability values, Transport in porous media, 53 (2003) 117-122.

[38] N.N. Anika, M.M. Hoque, Thermal buoyancy force effects on developed flow considering hall and ion-slip current, Annals of Pure and Applied Mathematics, 3 (2013) 179-188.

[39] N.N. Anika, M.M. Hoque, N. Islam, Hall current effects on magnetohydrodynamics fluid over an infinite rotating vertical porous plate embedded in unsteady laminar flow, Annals of Pure and Applied Mathematics, 3 (2013) 189-200.

[40] B. Mohanty, S. Mishra, H. Pattanayak, Numerical investigation on heat and mass transfer effect of micropolar fluid over a stretching sheet through porous media, Alexandria Engineering Journal, 54 (2015) 223-232.

[41] S. Mishra, G. Dash, P. Pattnaik, Flow of heat and mass transfer on MHD free convection in a micropolar fluid with heat source, Alexandria Engineering Journal, 54 (2015) 681-689.

[42] R. Tripathy, G. Dash, S. Mishra, S. Baag, Chemical reaction effect on MHD free convective surface over a moving vertical plate through porous medium, Alexandria Engineering Journal, 54 (2015) 673-679.

[43] M. Rashidi, S. Mohimanian, S. Abbasbandy, Analytic approximate solutions for heat transfer of a micropolar fluid through a porous medium with radiation, Communications in Nonlinear Science and Numerical Simulation, 16 (2011) 1874-1889.

[44] M. Muskat, R.D. Wyckoff, Flow of homogeneous fluids through porous media, (1937).

[45] R.E. Collins, Flow of fluids through porous materials, (1976).

[46] J. Bear, Dynamics of fluids in porous media, Courier Corporation2013. 